\title{
Evaluating the cost and clinical effectiveness of long- acting, injectable aripiprazole and paliperidone palmitate once a month in a real-world setting
}

This article was published in the following Dove Press journal:

ClinicoEconomics and Outcomes Research

\author{
Richard E Hodgson \\ Retiree, Staffordshire, UK
}

Correspondence: Richard E Hodgson North Staffordshire Combined Healthcare Trust, Lawton House, Bellringer Road, Stoke on Trent, ST4 $8 \mathrm{HH}, \mathrm{UK}$

Tel +44 I78 58I 8046

Email rhod819147@aol.com
Background: Antipsychotics are an effective treatment option for patients with schizophrenia and several long-acting injectables (LAIs), including risperidone, olanzapine, paliperidone, and aripiprazole are available in the UK. Treatment adherence is an issue in severe mental illness and LAIs have been hypothesized to offer clinical advantages over their oral equivalents. However, meta-analyses of randomized clinical trials and mirror-image studies have yielded equivocal results and have focused on economic rather than clinical outcomes. Objective: This study evaluated routinely collected data to ascertain cost-effectiveness and clinical outcomes of two LAIs, aripiprazole and paliperidone palmitate once a month. Clinical effectiveness was assessed using the Health of the Nation Outcome Scale (HoNOS) scores.

Materials and methods: A 1-year mirror image observational study of patients cared for by North Staffordshire Combined Healthcare Trust who received either the LAIs aripiprazole or paliperidone once a month from 2011 until the end of the study in 2016. The primary outcome was the effectiveness of the LAIs using a mirror-image design with HoNOS scores and bed use as the main outcomes.

Results: A total of 30 patients received aripiprazole and 84 received paliperidone once a month during the study period. A significant reduction in bed use was accompanied by clinical improvements, as measured by the HoNOS for both study drugs (aripiprazole: 13.4 before vs 8.7 after $[P=0.001]$; paliperidone: 15.8 before vs 12.9 after $[P=0.02]$ ). Analysis of several metabolic markers showed mean values within the normal range but the mean prolactin level in patients treated with paliperidone once a month was elevated.

Conclusion: The LAIs aripiprazole and paliperidone once a month are associated with clinical improvement and reduced bed use. Routine data gathering using, for example, HoNOS will offer the opportunity to provide additional insights into the clinical and cost benefits associated with LAIs and other interventions in the treatment of schizophrenia.

Keywords: schizophrenia, long-acting injectables, aripiprazole, paliperidone

\section{Introduction}

Schizophrenia is a disorder that affects $\sim 1$ in 100 UK adults. ${ }^{1}$ The International Classification of Diseases (10th revision) describes schizophrenia as a condition of "severe fundamental and characteristic distortions of thinking and perception, and affects that are inappropriate or blunted". Since the early 1950s, antipsychotic medication has been the primary treatment option for patients with schizophrenia. ${ }^{2}$

Antipsychotics are generally described as first generation (FGA) or second generation (SGA) and, while they vary in their receptor-binding profiles, pragmatically, these 
drugs have been defined by their side-effect profile. FGAs are generally associated with extrapyramidal adverse effects, with SGAs primarily associated with metabolic side effects.

Antipsychotics are an effective treatment for patients with schizophrenia and the mainstay of current clinical care. Long-acting injectable (LAI) antipsychotics offer an important alternative to oral treatment options in the context of medication adherence. Several SGA antipsychotics, such as risperidone, olanzapine, paliperidone palmitate, and aripiprazole, are approved in the UK for the treatment of schizophrenia in both oral and LAI preparations. Paliperidone and aripiprazole have been available as LAIs in the UK from 2011 and 2014, respectively but are more expensive compared with FGA LAI antipsychotics.

Results of placebo-controlled, registration trials of SGA LAIs demonstrated a significant reduction in relapse rates and improvement in symptoms compared with placebo in patients with chronic schizophrenia, as well as in acutely ill patients. ${ }^{3}$ Clinical trials comparing SGA LAIs with oral antipsychotics (OAPs) are less consistent, being influenced considerably by study design, with the superior efficacy of LAIs to OAPs most evident in mirror image and cohort studies. ${ }^{4,5}$

Poor medication adherence, as with other medical conditions, can be a significant issue in those with serious mental illnesses, with poor adherence usually associated with inferior clinical outcomes. For example, a study from the USA using Medicaid databases of patients with schizophrenia showed that those patients who were non-adherent were approximately three times more likely to be admitted to a psychiatric bed over a year than their adherent counterparts. ${ }^{6}$ Even when patients report satisfaction with their medication choice, there is evidence of under-adherence. Both patients and prescribers overestimate adherence rates, when adherence is measured electronically via a microchipped medication container. ${ }^{7}$ Improved adherence is likely to result in better clinical and patient-reported outcomes. LAIs, in contrast to oral formulations, can offer benefits in terms of convenience, increased availability of clinician resource, patient autonomy, and potentially enhanced adherence.

The first SGA LAI, risperidone, was launched in 2001. Currently, risperidone, paliperidone, olanzapine, and aripiprazole are approved for the treatment of patients with schizophrenia in the UK; however, the benefits of using LAI SGAs over oral SGAs are unclear. ${ }^{8}$ Meta-analyses have suggested that there is no firm evidence that atypical LAI antipsychotic medications reduce the overall relapse rate in patients with schizophrenia compared with their oral equivalents, although patients' global outcomes generally show improvement. ${ }^{5,8}$ The lack of apparent effectiveness may be due to some of the methodological limitations of randomized controlled trials (RCTs), including their shorter duration. This short duration may miss the benefits of medication over a longer time-period (eg, several years) from being observed. Furthermore, patients selected for RCTs are less likely to be those who will benefit most from LAI therapies, such as those who poorly engage with services. ${ }^{4,5}$

Observational studies have shown that LAIs, when compared with their oral equivalents, reduce relapse and readmission rates significantly. ${ }^{4,9}$ Mirror-image studies of antipsychotic LAIs provide an alternative model of study, in which each patient acts as their own control. These studies have the advantage of assessing real-world patients, and results suggest reduced relapse rates with LAI use. ${ }^{1}$ Usually, mirror-image studies focus on economic outcomes and do not report clinical outcomes.

The Health of the Nation Outcome Scale (HoNOS) is a validated scale that measures the health and social functioning of people with severe mental illness. ${ }^{11}$ It was developed in 1993 as a 12-item validated scale to measure behavior, impairments, symptoms, and social functioning, to be used as a routine clinical outcome measure. It is now the most widely used clinical outcome measure routinely used by global mental health services. Patients in England using psychiatric services are routinely evaluated using the HoNOS as part of mandatory Governmental health improvement initiatives.

The LAI form of aripiprazole (Abilify Maintena ${ }^{\circledR}$, Otsuka Pharmaceutical Europe Ltd., Slough, UK) is indicated for maintenance treatment of adult patients with schizophrenia stabilized with oral aripiprazole, and was approved in the UK in November 2013. Paliperidone palmitate LAI (Xeplion ${ }^{\circledR}$, Janssen-Cilag, Beerse, Belgium) is indicated for maintenance treatment of adult patients with schizophrenia stabilized with paliperidone or risperidone, and was approved in the UK in March 2011. Both have a higher acquisition cost compared with generic antipsychotics and therefore, local commissioners required an evaluation of their use. The primary objective of this study was to undertake a service evaluation using routinely collected data to ascertain both the cost effectiveness and clinical effectiveness of LAI aripiprazole and paliperidone once a month against HoNOS scores.

At initiation of the service evaluation, there was a lack of relevant literature. However, a part-prospective mirror-image study examining outcomes 2 years before starting paliperidone palmitate and 2 years after initiation of treatment has since been reported. This study demonstrated a substantial reduction in hospital admissions (from 1.80 in the 2 years 
before starting paliperidone once a month to 0.81 in the 2 years following initiation $(P<0.001))$ and days spent in hospital, which was associated with cost reductions related to reduced use of health care facilities. ${ }^{12}$ Further, a recent noninterventional, observational, mirror-image follow-up of 160 patients prescribed LAI aripiprazole at two UK centers showed significant reductions in the mean number of bed days in the year following LAI aripiprazole, from $\sim 30$ to 23 days per patient. The mean number of admissions also fell significantly from 0.71 to 0.45 per patient per year over the same period. The prescribing of LAI aripiprazole reduced average bed days and admissions compared with prior treatments and was viewed as broadly cost-neutral. ${ }^{13}$ Both these studies focused on economic outcomes and did not report specific clinical outcomes.

More data on the wider health and social impact of aripiprazole or paliperidone on patients with clinically diagnosed psychosis are needed. In this study, we used the validated HoNOS scale to measure improvements in wider health and functioning and as a secondary measure to review patients' metabolic parameters, given mounting concerns regarding the physical health of patients with psychosis.

\section{Materials and methods}

This study was conducted in North Staffordshire, UK; a mixed urban and rural area with a population of 470,000 , high levels of social deprivation, and limited geographical mobility. Secondary care adult mental health services are provided by one organization, North Staffordshire Combined Healthcare Trust (NSCHT), and include inpatient services, crisis/home treatment, mental health resource centers, and an early intervention service. Due to local agreements, all patients treated with LAIs are managed in secondary services.

All consecutive patients were identified from pharmacy records who had received either LAI aripiprazole or paliperidone once a month since their respective launches in the UK until the end of the study in 2016. The sample included all patients who received at least two consecutive doses of the LAI. Too few patients were prescribed long-acting olanzapine and risperidone to warrant their inclusion in the study. There were no inclusion or exclusion criteria. Patients receiving LAI aripiprazole were followed up for the 1-year mirror period, as paliperidone once a month was introduced earlier, the longer follow-up period was available. During the study period, there were no restrictions on the prescription of either LAI other than completing an offformulary form, which detailed clinical information, diagnosis, and why on formulary drugs were not suitable. There were no significant changes in bed numbers, patient numbers, or crisis/home treatments during the study period.

Admission and bed occupancy data were obtained from NSCHT's computerized inpatient management system; its reliability has been demonstrated previously. ${ }^{14}$ HoNOS data and some demographic features were obtained from electronic care program approach data. The HoNOS score just prior to initiation of each LAI was recorded, as was the score at 1 year (and 2 years for paliperidone once a month) follow-up. Patient records were examined for clinical details and blood results. Recommendations for physical health monitoring in the UK are varied, including serum lipids, glucose, and prolactin, but at a minimum, annual monitoring is recommended. Obtaining serum values prior to LAI initiation was not feasible and potentially misleading; values were recorded nearest to 1 -year follow-up. ${ }^{15}$

The primary outcome was a mirror-image analysis of total days of inpatient care in the 1 -year period prior to the administration of the LAI and the 1-year following first injection through an assessment of clinical and cost effectiveness. Cost analysis was based on LAI acquisition costs, as published in 2015 and did not include administration costs. Bed costs were also calculated for 2015, the midpoint of the follow-up period. All admissions were assumed to be assigned to a general psychiatry bed for costing calculations as it was not possible to accurately cost higher observation levels.

Analysis was based on an intention-to-treat basis. Data were analyzed using SPSS version 21 (IBM SPSS Statistics for Windows). The distribution of numbers of admissions and bed days was non-normal and Wilcoxon signed-rank test and bootstrap paired sample $t$-test were used to test the null hypothesis of no difference in number of admissions and bed days before and after initiating LAI.

The study was a service evaluation using routinely collected data negating the need for ethics committee approval.

\section{Results}

A total of 114 patients (30 on aripiprazole and 84 on paliperidone palmitate once a month) received a LAI drug during the study period. Overall, 65\% were male and the mean age was 40 years. Approximately 50\% were detained under 1983 Mental Health Act (MHA) when the LAIs were initiated. The median length of diagnosis was $>10$ years.

A total of 30 patients received LAI aripiprazole during the study period; patient characteristics are shown in Table 1. Most of these patients were single, male, and white. The median dose of LAI aripiprazole was $400 \mathrm{mg}$ with only one patient receiving $300 \mathrm{mg}$. Most patients were started on LAI 
Table I Patient characteristics at start of study into cost and clinical effectiveness of LAI aripiprazole and paliperidone once a month

\begin{tabular}{|c|c|c|}
\hline & \multicolumn{2}{|c|}{ Baseline characteristics } \\
\hline & $\begin{array}{l}\text { Aripiprazole } \\
(n=30)\end{array}$ & $\begin{array}{l}\text { Paliperidone } \\
(n=84)\end{array}$ \\
\hline Gender, male n (\%) & $21(70)$ & $56(67)$ \\
\hline Mean age, years (SD) & $39(14.1)$ & $41(13.5)$ \\
\hline Marital status, single $n(\%)$ & $26(87)$ & $55(65)$ \\
\hline \multicolumn{3}{|l|}{ Ethnicity, n (\%) } \\
\hline White & $25(83.3)$ & $71(84.0)$ \\
\hline Indian & $2(6.7)$ & $2(2.4)$ \\
\hline African & $\mathrm{I}(3.3)$ & $3(3.6)$ \\
\hline Afro-Caribbean & $2(6.7)$ & $2(2.4)$ \\
\hline Other & 0 & $6(7.1)$ \\
\hline Length of diagnosis, months (SD) & $120.8(83.6)$ & $132.0(69.0)$ \\
\hline Median number of previous admissions & 5 & 5 \\
\hline Treatment initiation inpatient, $\mathrm{n}(\%)$ & $21(70)$ & $60(7 \mathrm{I})$ \\
\hline Mental Health Act status, \% detained & 53.3 & 53.6 \\
\hline Reasons for starting LAl treatment, $\mathrm{n}(\%)$ & & \\
\hline Lack of effectiveness/poor adherence & $25(84)$ & $72(86)$ \\
\hline Prior clozapine & I (3.3) & $7(8.3)$ \\
\hline
\end{tabular}

Abbreviation: LAI, long-acting injectable.

aripiprazole in an inpatient setting, with 9 (30\%) started while on a psychiatric intensive care unit. A total of 16 (53.3\%) patients were detained under the 1983 MHA when LAI aripiprazole was started. Of the 30 patients, $3(10 \%)$ patients had not received other antipsychotics before starting LAI aripiprazole treatment, and one patient had participated in a previous trial of clozapine. The median number of admissions prior to LAI aripiprazole therapy was five and the most common reason for starting LAI aripiprazole therapy was poor adherence with previous treatment (13 patients) and lack of prior treatment efficacy (11 patients). Of those receiving LAI aripiprazole, 4 (13.3\%) were switched due to side effects with previous medication, while for $2(6.6 \%)$, the reason for switching/initiation was unclear. In the follow-up period, side effects were documented for four patients, although it was difficult to assess as to the severity or persistence of these from the available notes. A total of 4 patients received anti-cholinergics, however, these had been initiated prior to LAI aripiprazole initiation in this study.

A total of 84 patients received LAI paliperidone once a month during the study period, at a mean dose of $106 \mathrm{mg}$; patient characteristics are shown in Table 1. Baseline characteristics were comparable with those receiving LAI aripiprazole with most patients also single, male, and white. The majority of patients (71\%) were started on LAI paliperidone once a month in an inpatient setting, with 24 patients (29\%) receiving medication while on a psychiatric intensive care unit (A unit with higher staffing and security levels). Upon LAI paliperidone once-monthly initiation, 45 (54\%) patients were detained under the 1983 MHA. The median number of admissions prior to LAI paliperidone oncemonthly therapy was five and the most common reason for starting therapy was lack of effectiveness/poor adherence with previous treatment (72 patients; $86 \%$ ). Of those receiving LAI paliperidone once a month, $7(8.3 \%)$ had previously received clozapine. For both LAIs, the most common previously prescribed antipsychotic was olanzapine. Given the clinical history of these patients, the majority had received multiple trials of antipsychotics prior to starting the respective LAIs.

Table 2 summarizes the main study outcomes; both bed occupancy and number of admissions were significantly lower with LAI aripiprazole and paliperidone once a month. The cost of LAI aripiprazole at the mean study dose was $£ 2,645$, whereas the cost of LAI paliperidone once a month for a 1 -year period was $£ 3,796$. The reduction in bed use equates to a minimum saving of $£ 14,175$ and $£ 13,750$ per patient with LAI aripiprazole and LAI paliperidone once a month, respectively. These calculations are based on the basic hotel bed cost and drug acquisition costs in 2015, which was the midpoint of the study period. The cost of the intensive care component could not be reliably estimated. However, it is hypothesized that the actual cost-saving in a routine clinical setting would be significantly higher. The reduction in bed use was accompanied by clinical improvements as measured by the HoNOS. The only sub-score not to show improvement with LAI aripiprazole was the impairment subscale, and the clinical notes recorded an improvement in $24(80 \%)$ patients. For LAI paliperidone once a month, a similar trend was seen in the clinical improvements, as measured by HoNOS.

More patients were receiving LAI paliperidone once a month given that it had been available in the UK for a longer period of time. This allowed for a 2-year mirage analysis for bed occupancy data, with similar results to 1 year. However, HoNOS data were not available for the first 26 LAI paliperidone once-monthly patients.

Blood results for $25 \mathrm{LAI}$ aripiprazole and $65 \mathrm{LAI}$ paliperidone once-monthly patients were analyzed for key metabolic markers (Table 3). Results taken at least 6 
Table 2 Principal outcomes seen for patients before and after initiating therapy with LAI aripiprazole or paliperidone once a month

\begin{tabular}{|c|c|c|c|}
\hline & Before & After & $P$-value \\
\hline \multicolumn{4}{|c|}{ Bed days, mean (SD) } \\
\hline Aripiprazole & $63.0(54.8)$ & $6.3(16.9)$ & 0.0001 \\
\hline Paliperidone & $69.0(80.0)$ & $13.9(36.0)$ & 0.001 \\
\hline \multicolumn{4}{|c|}{ Admissions, mean (SD) } \\
\hline Aripiprazole & $\mathrm{I} .64(\mathrm{I} .5)$ & $0.46(0.9)$ & 0.0001 \\
\hline Paliperidone & $\mathrm{I} .33(0.9)$ & $0.59(1.6)$ & 0.001 \\
\hline \multicolumn{4}{|c|}{ Total HoNOS score, mean (SD) } \\
\hline Aripiprazole & I $3.4(6.4)$ & $8.7(5.4)$ & 0.001 \\
\hline Paliperidone $^{\mathrm{a}}$ & $15.8(7.6)$ & $12.9(8.4)$ & 0.02 \\
\hline \multicolumn{4}{|c|}{ Behavior sub-score, mean (SD) } \\
\hline Aripiprazole & $2.3(2.0)$ & I.I (I.5) & 0.013 \\
\hline Paliperidone & $3.0(2.2)$ & $2.2(2.0)$ & 0.02 \\
\hline \multicolumn{4}{|c|}{ Symptoms sub-score, mean (SD) } \\
\hline Aripiprazole & $4.6(2.7)$ & $2.6(2.2)$ & 0.002 \\
\hline Paliperidone & $5.4(2.6)$ & $3.9(2.6)$ & 0.02 \\
\hline \multicolumn{4}{|c|}{ Impairments sub-score, mean (SD) } \\
\hline Aripiprazole & $0.8(1.4)$ & $0.9(1.5)$ & 0.839 \\
\hline Paliperidone & $1.69(1.8)$ & $1.53(1.8)$ & 0.421 \\
\hline \multicolumn{4}{|c|}{ Social sub-score, mean (SD) } \\
\hline Aripiprazole & $5.7(3.6)$ & $4.1(3.9)$ & 0.035 \\
\hline Paliperidone & $5.5(4.3)$ & $4.2(4.4)$ & 0.03 \\
\hline
\end{tabular}

Notes: ${ }^{a}$ HoNOS score available for 58 patients (see "Results" section).

Abbreviations: HoNOS, Health of the Nation Outcome Scale; LAI, long-acting injectable.

Table 3 Key metabolic markers from blood tests for patients participating in study

\begin{tabular}{|c|c|c|c|c|c|c|}
\hline & Cholesterol & HDL & LDL & Triglyceride & Glucose & Prolactin \\
\hline Normal range & $<5 \mathrm{mmol} / \mathrm{L}$ & $\mathrm{I}-\mathrm{I} .5 \mathrm{mmol} / \mathrm{L}$ & $<3 \mathrm{mmol} / \mathrm{L}$ & $<1.7 \mathrm{mmol} / \mathrm{L}$ & $4.4-7.8 \mathrm{mmol} / \mathrm{L}$ & $\begin{array}{l}\text { Males: }<425 \mathrm{mlU} / \mathrm{L} \\
\text { Non-gravid females: } 106-850 \mathrm{mlU} / \mathrm{L}\end{array}$ \\
\hline Mean (SD) & & & & & & \\
\hline Aripiprazole & 4.67 (I.18) & I.28 (0.33) & 2.51 (0.89) & $1.76(1.02)$ & $5.23(1.35)$ & $254.90(317.69)$ \\
\hline Paliperidone & $4.84(1.03)$ & $1.39(0.47)$ & $2.66(0.92)$ & $1.59(0.92)$ & $5.42(1.90)$ & 954.11 (74I.52) \\
\hline
\end{tabular}

Abbreviations: HDL, high-density lipoprotein; LDL, low-density lipoprotein.

months after the initiation of the LAI, and the mean values for all measurements lie within the normal range expected for these parameters, with the exception of the mean prolactin level in patients treated with paliperidone 954 $\mathrm{mIU} / \mathrm{L}$, which was above the normal range, and higher than that seen with aripiprazole $255 \mathrm{mIU} / \mathrm{L}$.

\section{Discussion}

The patient characteristics seen at the start of the study were skewed toward a potentially more difficult-to-treat group, as judged by the median number of previous admissions. The results show reduced bed occupancy, reduced associated costs, and an improvement in symptoms and social functioning on the HoNOS for both LAIs. This was mirrored by clinical teams who reported that $\sim 80 \%$ of patients showed a functional improvement. However, there was no improvement in the impairment subscale of the HoNOS scores with either LAI, which is a common finding. ${ }^{16}$ This lack of improvement may demonstrate longstanding, relatively intractable problems, including negative symptoms, which may not be as responsive to pharmacotherapy as positive symptoms. ${ }^{17}$ 
A statistical analysis was not performed to determine whether there were significant differences between the two LAIs due to the data being mostly non-contemporaneous due to the earlier availability of LAI paliperidone once a month than LAI aripiprazole. To the best of our knowledge, there is only one head-to-head comparison of LAI aripiprazole and LAI paliperidone once a month. This randomized, non-inferior, open-label, rater-blinded study was conducted $>28$ weeks in 295 patients with schizophrenia. The study showed that $68 \%$ of aripiprazole and $57 \%$ of paliperidone patients completed 28 weeks of treatment. Statistically significant improvements in clinician-rated, health-related, and quality of life scores were seen with aripiprazole-treated patients. ${ }^{18}$ Further, a sub-analysis in younger patients $<35$ years of age showed improvement in outcomes for LAI aripiprazole. ${ }^{18}$

Overall, the results of this small, real-world study are in accordance with other studies, and support switching patients from oral to LAI antipsychotics. ${ }^{19-21}$ These results suggest considerable cost savings as a result of reduced bed use in patients treated with LAI aripiprazole over a period of 1 year. These data are in contrast to Taylor et al (2016) where the use of LAI aripiprazole is described as cost neutral. ${ }^{13}$ It has also been reported in a 2-year mirror study that paliperidone LAI was cost effective. ${ }^{12}$

Individual blood results were collected $\sim 6-12$ months after treatment was initiated. No attempt was made to compare these with results prior to the prescription of LAI treatment, as there would be a chance of confounding based on the level of adherence and prior antipsychotic used, thus limiting interpretation of the results. Interestingly, the changes in prolactin levels observed with LAI paliperidone once a month have also been noted in other RCTs where $\sim 3 \%$ of patients had prolactin-related adverse events. ${ }^{22}$

Cross-sectional data allow the comparison of results with shorter RCTs. ${ }^{23}$ From a clinical point of view, at the 1-year follow-up, a cross-sectional view of the blood results from all patients demonstrated no major differences in results from short-term use of either antipsychotic in oral or LAI preparation. This small sample and 1-year follow-up may not reveal all long-term problems seen with LAI use, but it is reassuring that no abnormalities were seen to date.

While antipsychotics are associated with side effects and abnormal laboratory tests results, ${ }^{23}$ evidence from short-term studies do not always reflect the evidence of longer term observational studies, which show that antipsychotic medication reduces morbidity rates. ${ }^{9,24}$ Gilmer et al (2004) demonstrated that medication adherence was not only associated with reductions in psychiatric admissions, but also general hospital admissions. ${ }^{6}$

There are several limitations with the current study. Despite RCTs seen as the "gold standard" for evaluating treatment efficacy, they are not without their disadvantages. ${ }^{25}$ Observational studies, including mirrorimage studies, inform real-world practice and better reflect LAI use in routine clinical practice. ${ }^{4}$ In a mirror-image study, patients act as their own control with no parallel placebo or active control group. The absence of a control group means strict comparison between aripiprazole and paliperidone are not reliable and only limited inferences can be made. The advantage is that "real-time change" against individual previous experience can be measured. However, the results may be subject to bias due to regression toward the mean or other non-controlled factors, such as more frequent contact with healthcare professionals. It is inferred from the data that the improvement in outcomes is due to enhanced medication adherence but our data cannot prove this.

One factor that may limit the generalizability of our results is the use of MHA Law to mandate hospital admission in approximately half the patients. It is a moot point as to how many would have accepted LAI treatment or indeed any form of antipsychotic if compulsory treatment was not mandated by law. Provision for compulsory community treatment following hospital discharge was available and was used for eight patients. Other limitations include the study sample being opportunistic, small, and limited by prescribing practices of local consultants, which may have introduced further bias. However, the chronicity and number of previous admissions suggest that this sample is a "hard-to-treat" group and the results may have been more favorable in a potentially easierto-treat group, such as younger patients. ${ }^{18}$ The sample is also more likely to reflect patients typically encountered in realworld clinical settings than those in RCTs. ${ }^{4,25}$

During the study period, no LAI patients received outof-area inpatient care. Many patients were started on LAI on a high observation level and/or on a psychiatric intensive care setting and it was not possible to reliably capture the extra or additional costs; therefore, it is likely that our results minimize potential cost savings. In addition, approaches that attribute bed days from an index admission to post-treatment immediately after initiation are likely to underestimate the positive effects of the new antipsychotic. $^{26,27}$ 
Routinely collected HoNOS scores were used to ascertain whether LAI aripiprazole and paliperidone once a month produced clinical improvements in parallel with reduced inpatient bed occupation. All clinicians had training in the use of this instrument, but there was no standardization and training to the same level as conducted in an RCT. However, the results support previously rigorously conducted research in North Staffordshire using the HoNOS both in terms of the magnitude of change as well as the impairments scale being refractory to improvement. ${ }^{16}$ This study does demonstrate that there is an opportunity to evaluate services more closely. Previous similar research with antipsychotic medications, such as LAIs, has focused on economic outcomes, such as occupied bed days, ${ }^{20}$ but the use of the HoNOS score demonstrates that this is not at the expense of clinical outcomes in the current study. Three out of the four HoNOS subscales improved, but the impairment scale did not (which is similar to previously reported findings). There is the potential that these outcomes may not be amenable to psychotropic treatment. However, a significant improvement in mental state should encourage people to engage with psychosocial activities designed to achieve this. As a secondary finding, there was no significant evidence of deterioration in the metabolic parameters of patients when using either LAI. The study also supports previously reported increases in mean prolactin levels seen with paliperidone once a month.

\section{Conclusion}

This study demonstrates that LAI aripiprazole and LAI paliperidone use once a month is associated with clinical improvement, which is reflected in reduced bed use. The reduction in bed use more than offsets the acquisition costs of LAI drugs, with cost savings in this study being larger for LAI aripiprazole than LAI paliperidone over a period of 1 year. Previous studies have demonstrated that atypical LAIs reduce hospital readmission rates, but this study further demonstrated that this is not at the expense of clinical and social outcomes for both aripiprazole and paliperidone. Furthermore, the clinical utility of routine outcomes, which are underused in the National Health Service (NHS) despite increasing demands on clinicians to collect data demonstrating improvements in the quality of care. Reassuringly, there were no discernible mean abnormalities in patient biochemical markers with either drug, with the exception of raised prolactin levels with LAI paliperidone. Analysis of routinely collected data on a regular basis within the NHS should be used to inform clinical practice and provide justification for the significant costs in generating these data.

\section{Acknowledgments}

The author would like to thank C Aladakatti, B Davenport, and T Saravanappa for their assistance with data collection and A Kinmond for help with manuscript revision. An unrestricted educational grant for manuscript writing and editing was provided by Lundbeck, with medical writing assistance provided by D Whitford and HAVAS Just. Lundbeck had no influence or input in this service evaluation and submission of manuscript.

\section{Disclosure}

Dr Hodgson has received lecture fees and educational support from Janssen, Lundbeck, and Otsuka. The author reports no other conflicts of interest in this work.

\section{References}

1. Semple D, Smyth R. Oxford Handbook of Psychiatry. 3rd ed. Oxford: Oxford University Press; 2013.

2. Hodgson RE, Lewis M, Boardman AP. Prediction of readmission to acute psychiatric units. Soc Psychiatry Psychiatr Epidemiol. 2001;36 (6):304-309.

3. Correll CU, Citrome L, Haddad PM, et al. The use of long-acting injectable antipsychotics in schizophrenia: evaluating the evidence. $J$ Clin Psychiatry. 2016;77(suppl 3):1-24.

4. Kishimoto T, Nitta M, Borenstein M, Kane JM, Correll CU. Longacting injectable versus oral antipsychotics in schizophrenia: a systematic review and meta-analysis of mirror-image studies. $J$ Clin Psychiatry. 2013;74(10):957-965

5. Kishimoto T, Robenzadeh A, Leucht C, et al. Long-acting injectable vs oral antipsychotics for relapse prevention in schizophrenia: a metaanalysis of randomized trials. Schizophr Bull. 2014;40(1):192-213.

6. Gilmer TP, Dolder CR, Lacro JP, et al. Adherence to treatment with antipsychotic medication and health care costs among Medicaid beneficiaries with schizophrenia. Am J Psychiatry. 2004;161(4):692-699.

7. Byerly MJ, Thompson A, Carmody T, et al. Validity of electronically monitored medication adherence and conventional adherence measures in schizophrenia. Psychiatr Serv. 2007;58(6):844-847.

8. Adams CE, Fenton MK, Quraishi S, David AS. Systematic metareview of depot antipsychotic drugs for people with schizophrenia. $\mathrm{Br}$ J Psychiatry. 2001;179(04):290-299.

9. Tiihonen J, Mittendorfer-Rutz E, Majak M, et al. Real-world effectiveness of antipsychotic treatments in a nationwide cohort of 29823 patients with schizophrenia. JAMA Psychiatry. 2017;74(7):686-693.

10. Schooler NR. Relapse and rehospitalization: comparing oral and depot antipsychotics. J Clin Psychiatry. 2003;64(Suppl 16):S14-S17.

11. Wing JK, Beevor AS, Curtis RH, et al. Health of the nation outcome scales (HoNOS). Research and development. $\mathrm{Br} J$ Psychiatry. 1998;172:11-18.

12. Taylor DM, Sparshatt A, O'Hagan M, Dzahini O. Effect of paliperidone palmitate on hospitalisation in a naturalistic cohort - a fouryear mirror image study. Eur Psychiatry. 2016;37:43-48.

13. Taylor DM, Sparshatt A, Amin F, et al. Aripiprazole long-acting injection - a mirror image study of its effects on hospitalisation at one year. J Psychopharmacol. 2017;31(12):1564-1569. 
14. Hodgson re, Lewis M, Boardman AP. The prediction of in-patient length of stay for acute psychiatric admissions. $J$ Ment Health. 2000;9(2):145-153.

15. Hodgson R, Adeyemo O. Physical examination performed by psychiatrists. Int J Psychiatry Clin Pract. 2004;8(1):57-60.

16. Boardman AP, Hodgson RE, Lewis M, Allen K. North Staffordshire community beds study: longitudinal evaluation of psychiatric inpatient units attached to community mental health centres. I: methods, outcome and patient satisfaction. Br J Psychiatry. 1999;175 (01):70-78.

17. Leucht S, Cipriani A, Spineli L, et al. Comparative efficacy and tolerability of 15 antipsychotic drugs in schizophrenia: a multipletreatments meta-analysis. Lancet. 2013;382(9896):951-962.

18. Naber D, Hansen K, Forray C, et al. Qualify: a randomized head-tohead study of aripiprazole once-monthly and paliperidone palmitate in the treatment of schizophrenia. Schizophr Res. 2015;168(12):498-504.

19. Haddad PM, Taylor M, Niaz OS. First-generation antipsychotic longacting injections $\mathrm{V}$. oral antipsychotics in schizophrenia: systematic review of randomised controlled trials and observational studies. $\mathrm{Br} \mathrm{J}$ Psychiatry. 2009;52:S20-28.

20. Kane JM, Sanchez R, Zhao J, et al. Hospitalisation rates in patients switched from oral anti-psychotics to aripiprazole once-monthly for the management of schizophrenia. J Med Econ. 2013;16(7):917-925.
21. Spill B, Konoppa S, Kissling W, et al. Long-term observation of patients successfully switched to risperidone long-acting injectable: a retrospective, naturalistic 18-month mirror-image study of hospitalization rates and therapy costs. Int J Psychiatry Clin Pract. 2010;14 (1):53-62

22. Gopal S, Vijapurkar U, Lim P, et al. A 52-week open-label study of the safety and tolerability of paliperidone palmitate in patients with schizophrenia. J Psychopharmacol. 2011;25(5):685-697.

23. Newcomer JM. Second-generation (atypical) antipsychotics and metabolic effects: a comprehensive literature review. CNS Drugs. 2005;19(Suppl 1):S1-S93.

24. Strom BL, Eng SM, Faich G, et al. Comparative mortality associated with ziprasidone and olanzapine in real-world use among 18,154 patients with schizophrenia: the ziprasidone observational study of cardiac outcomes (ZODIAC). Am J Psychiatry. 2011;168(2):193-201.

25. Hodgson R, Bushe C, Hunter R. Measurement of long-term outcomes in observational and randomised controlled trials. $\mathrm{Br} J$ Psychiatry. 2007;50(Suppl):S78-S84.

26. Faries DE, Nyhuis AW, Ascher-Svanum H. Methodological issues in assessing changes in costs pre- and post-medication switch: a schizophrenia study example. Cost Eff Resour Alloc. 2009;7(1):11.

27. Taylor D, Olofinjana O. Long-acting paliperidone palmitate - interim results of an observational study of its effect on hospitalization. Int Clin Psychopharmacol. 2014;29(4):229-234.
ClinicoEconomics and Outcomes Research

\section{Publish your work in this journal}

ClinicoEconomics and Outcomes Research is an international, peerreviewed open-access journal focusing on Health Technology Assessment, Pharmacoeconomics and Outcomes Research in the areas of diagnosis, medical devices, and clinical, surgical and pharmacological intervention. The economic impact of health policy and health systems organization also constitute important areas of coverage. The manuscript management system is completely online and includes a very quick and fair peer-review system, which is all easy to use. Visit http://www.dovepress.com/testimonials.php to read real quotes from published authors. 